\title{
ON EXPERIMENTAL DATA OF THE TCR OF TFRS AND THEIR RELATION TO THEORETICAL MODELS OF CONDUCTION MECHANISM
}

\author{
I. STORBECK and M. WOLF \\ Zentralinstitut für Festkörperphysik und Werkstofforschung der Akademie der Wissenschäften der \\ DDR, Dresden ${ }^{\dagger}$
}

(Received 25 May 1983; in final form 5 October 1983)

\begin{abstract}
Any theory of electrical conduction in TFRs encounters mainly two problems: (i) explanation of the dependence of $R_{\square}$ on properties of conducting component (volume fraction, grain size, resistivity), (ii) explanation of the temperature dependence of $R_{\square}$ taking into account (i). In order to achieve this one has to fit some microscopic parameters to experimental $R_{\square}$-and TCR-values, and to check if they are reasonable or not. The aim of the following discussion is to show, that such a fitting by means of experimental TCR-values is not correct. This is due to the fact that TCR-behaviour, as is well known, is determined also by the dependence of resistivity on strain. But any theoretical model neglects strains, also those who are induced by thermal strains. By means of published experiments concerning the strain dependence of resistance, the magnitude is estimated by which the TCR-values have to be corrected for the described fit.
\end{abstract}

\section{INTRODUCTION}

One of the most important properties, that any theory of TFRs has to explain, is their very low TCR at ambient temperatures. A lot of experimental results and their theoretical analysis led to the conclusion, that tunnelling (modified, because of trap-activation and the small size of metallic-like grains) through glassy interlayers determines the resistivity of TFRs. ${ }^{1,2}$ It is also well known, that TFRs exhibit a large piezo-resistive effect. ${ }^{3}$ The aim of this paper is to show, that because of the piezo-resistive effect, the measured TCR-values have to be corrected by a term, determined by strain dependence of the resistivity, if they are to be compared with theoretical models or are to be used to fit any parameter in such models. This is necessary, because in any theoretical model thermal expansion of TFR is neglected.

\section{THEORETICAL CONSIDERATIONS}

From experiments it is well known that TFRs exhibit a large piezo-resistive effect. ${ }^{3}$ This means that the resistance $R$ increases if a strain $\epsilon$ is applied. Let us describe this phenomenologically by:-

$$
\mathrm{R}=\rho(\mathrm{T}, \epsilon) \cdot \frac{1}{\mathrm{bd}},
$$

where $\rho$ denotes the resistivity dependent on temperature $\mathrm{T}$ and strain $\epsilon . \quad 1, \mathrm{~b}, \mathrm{~d}$ are the length, width, and thickness of the TFR, respectively. $\ddagger$ Note that we have assumed the

\footnotetext{
tDDR - 8027 Dresden, Postfach

${ }^{\ddagger}$ Experimentally a non-linear dependence of $\mathbf{R}$ on 1 is observed. These effects are due to interaction with contacts (constriction resistance, migration of metallic particles). This means different values of 1 correspond to different physical systems. This influence has to be suppressed. Hence the estimations in the following have to be based on (1).
} 
resistivity $\rho$ to be a function of $\epsilon$, not of stress. This is appropriate because in theoretical models $\epsilon=$ constant is assumed.

If the temperature is changed, one has to take into account that $\mathrm{R}$ changes also, because TFR (called film in the following) and substrate expand. That means, $\epsilon$ and also $\mathrm{l}, \mathrm{b}, \mathrm{d}$ are temperature-dependent. If one defines by

$$
\mathrm{TC} \rho=\left.\frac{1}{\rho} \quad \partial \rho\right|_{\epsilon=\text { const }}
$$

the temperature coefficient of $\rho$ under the condition $\epsilon=$ constant, the theoretical analysis for the TCR gives:

$$
\begin{aligned}
\mathrm{TCR} & =\frac{1}{\mathrm{R}} \frac{\mathrm{dR}}{\mathrm{dT}}=\mathrm{TC} \rho+\frac{1}{\rho} \frac{\partial \rho}{\partial \epsilon}\left[2 \alpha_{\mathrm{s}}+\alpha_{\mathrm{f}}-\frac{2 \nu_{\mathrm{f}}}{1-\nu_{\mathrm{f}}}\left(\alpha_{\mathrm{s}}-\alpha_{\mathrm{f}}\right)\right] \\
& +\frac{2 \nu_{\mathrm{f}}}{1-\nu_{\mathrm{f}}}\left(\alpha_{\mathrm{s}}-\alpha_{\mathrm{f}}\right)-\alpha_{\mathrm{f}} .
\end{aligned}
$$

Eqn. (2) holds for thin films, if these do not influence the expansion of the substrate. In typical cases $\mathrm{d} \approx 10 \mu \mathrm{m}$, but the thickness of the substrate is of the order of $1 \mathrm{~mm}$, and thus Eqn. (2) is applicable. In (2) $\alpha_{\mathrm{s}}, \alpha_{\mathrm{f}}$ denote the coefficients for thermal expansion of the substrate and film respectively. $\nu_{\mathrm{f}, \mathrm{s}}$ denotes the Poisson modulus of the film (substrate). The second term arises from the temperature-dependence of $\epsilon$, the last two from the temperature-dependence of $1, b$, and $d$.

Information concerning $\rho^{-1} \cdot \partial \rho / \partial \epsilon$ can be obtained by the measurement of the gauge factor. ${ }^{3}$ The longitudinal gauge factor, $\mathrm{GF}_{\mathrm{L}}=\mathrm{R}^{-1} \cdot \mathrm{dR} / \mathrm{d} \epsilon$, describes the relative change of resistance, if a strain $\epsilon$ is applied to substrate and the current is parallel to this component of strain. The following relation holds

$$
\frac{\mathrm{GF}_{\mathrm{L}}-1-\nu_{\mathrm{s}}}{1-\nu_{\mathrm{s}}}=\frac{1}{\rho} \frac{\partial \rho}{\partial \epsilon} \cdot \frac{1-2 \nu_{\mathrm{f}}}{1-\nu_{\mathrm{f}}}+\frac{\nu_{\mathrm{f}}}{1-\nu_{\mathrm{f}}}
$$

The gauge factor depends on both the Poisson moduli, $\nu_{\mathrm{f}}$ and $\nu_{\mathrm{s}}$, because there is a strain in the d-direction (determined by $\nu_{\mathrm{f}}$ and $\nu_{\mathrm{s}}$ ) and a strain, $-\nu_{\mathrm{s}} \epsilon$, in the b-direction, if $\epsilon$ is applied in the 1-direction.

By means of (2) and (3) it is possible to estimate the quantity $\mathrm{TC} \rho$, the temperature coefficient of the resistivity under the condition $\epsilon=$ const., if one takes into account the following:-

i) $\alpha_{\mathrm{s}}$ is known, $\alpha_{\mathrm{s}} \approx 6.1 \cdot 10^{-6} \mathrm{~K}^{-1}$ for $\mathrm{Al}_{2} \mathrm{O}_{3}$-substrates;

ii) $\alpha_{f}$ has been measured for different pastes and depends on $R_{\square}$. For $B_{2} R_{2} \mathrm{O}_{7}$ based TFRs a value of $\alpha_{\mathrm{f}}=(6.9 \ldots 8) \cdot 10^{-6} \mathrm{~K}^{-1}$ has been obtained

iii) $\nu_{\mathrm{s}}=0.2$;

iv) no information as regards $\nu_{\mathrm{f}}$ could be obtained. However by well-known physical reasons $\nu_{\mathrm{f}}$ obeys the relation $0 \leqslant \nu_{\mathrm{f}} \leqslant 0.5 .^{8}$ Within these limits the two expressions $\left(1-2 \nu_{\mathrm{f}}\right) /\left(1-\nu_{\mathrm{f}}\right)$ and $\nu_{\mathrm{f}} /\left(1-\nu_{\mathrm{f}}\right)$ in (3) decrease and increase, respectively, monotonically with $\nu_{\mathrm{f}}$. Therefore the right-hand side of (3), which is a linear function of $\rho^{-1} \partial \rho / \partial \epsilon$, behaves as shown in Figure 1. Because, for TFRs, the left-hand side of (3) is larger than 1, from Figure 1 it follows immediately that:-

$$
\frac{1}{\rho} \frac{\partial \rho}{\partial \epsilon}>\left.\frac{1}{\rho} \frac{\partial \rho}{\partial \epsilon}\right|_{\min }=\frac{\mathrm{GF}_{\mathrm{L}}-1-\nu_{\mathrm{s}}}{1-\nu_{\mathrm{s}}}
$$




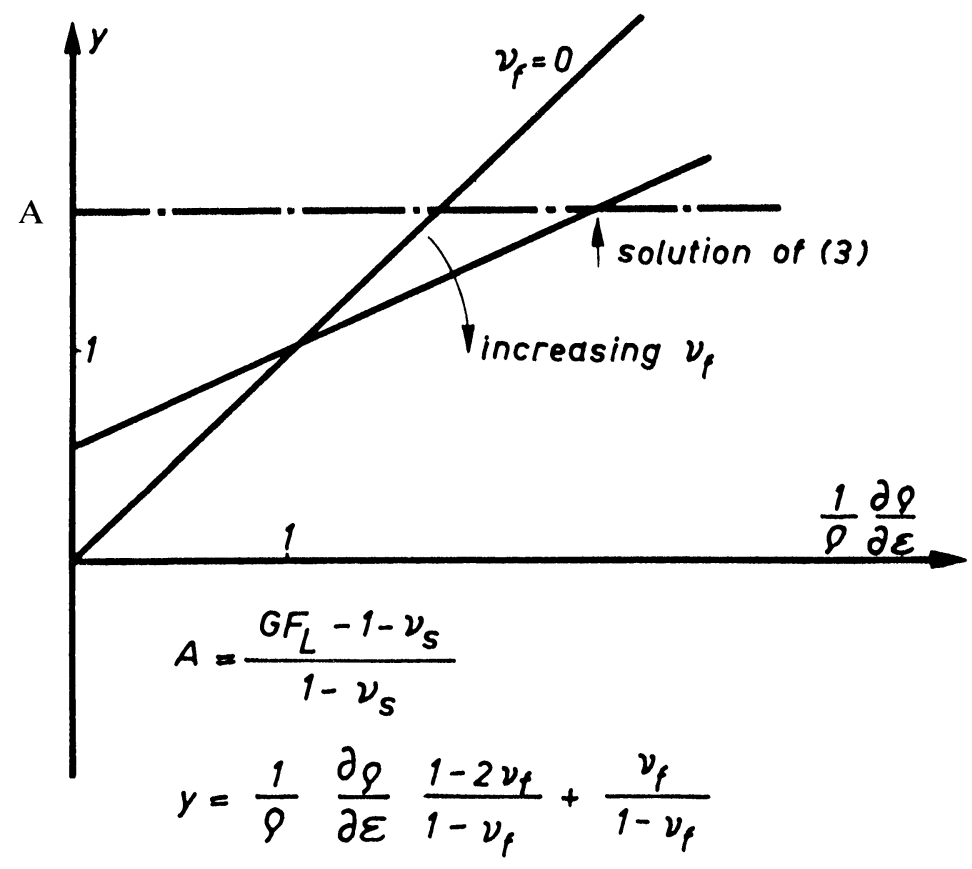

FIGURE 1 Behaviour of the solution of eqn (3) with increasing $v_{\mathbf{f}}$

holds. Thus in a rough approximation $\rho^{-1} \cdot \partial \rho / \partial \epsilon \approx \mathrm{GF}_{\mathrm{L}}$, and for the second term in (3) a value of about $180 \mathrm{ppm} \mathrm{K}{ }^{-1}$ is expected $\left(\mathrm{GF}_{\mathrm{L}} \approx 10\right.$ typically). Therefore, neglecting the difference between $\alpha_{\mathrm{f}}$ and $\alpha_{\mathrm{s}}$ and dropping the last two terms in (3) (which are of the order of some $10^{-6} \mathrm{~K}^{-1}$ ) result in an error smaller than $5 \%$ for typical TCR values of about $(50 \ldots 100) \cdot 10^{-6} \mathrm{~K}^{-1}$. Within these approximations TC $\rho$ can be estimated by:-

$$
\mathrm{TC} \rho \leqslant\left.\mathrm{TC} \rho\right|_{\max }=\mathrm{TCR}-\frac{\mathrm{GF}_{\mathrm{L}}-1-\nu_{\mathrm{s}}}{1-\nu_{\mathrm{s}}} 3 \alpha_{\mathrm{s}}
$$

The results are given in the next section. It contains also the results for $\mathrm{TC} \rho$ assuming $\nu_{\mathrm{f}}=\nu_{\mathrm{s}}$. This assumption is reasonable, because a TFR contains a lot of glass. These values have been calculated to test the sensitivity of our estimations.

\section{ESTIMATION OF TC $\rho$ FOR DU PONT 1441 AND DU PONT 1451 PASTES}

In this section the results of the estimations for the temperature coefficient of the thickfilm material TC $\rho$ are given for the pastes DP 1441 and DP 1451. The TCR-values for these pastes have been published by C. Canali et al. ${ }^{4}$, and A. Cattaneo and M. Prudenziati. ${ }^{5}$ Values of the gauge factors have been published by C. Canali et al. ${ }^{3}$ Tables I and II present the measured TCR, TC $\left.\rho\right|_{\text {max. }}$. (estimated by means of (5)) and $\left.\mathrm{TC} \rho\right|_{\boldsymbol{\nu} \mathbf{f}=\nu_{\mathbf{s}}}$ (if $\nu_{\mathrm{f}}=\nu_{\mathrm{s}}$ holds) at different temperatures. Because of the narrow temperature range considered here, $\alpha_{\mathrm{f}}, \alpha_{\mathrm{s}}, \nu_{\mathrm{s}}$ can be assumed to be constant. Moreover an isotropic thermoelastic behaviour has been assumed. Because terms of the order $10^{-6} \cdot \mathrm{K}^{-1}$ have been neglected, all results are given in an approximated form. Here it should be pointed out once again, that $\mathrm{TC} \rho$-values in the third line are the highest possible ones. 


\section{DISCUSSION}

From the results, given in Table I and Table II, the following can be concluded:

1) The small TCR of TFRs at ambient temperatures $\left(-30 \ldots+120^{\circ} \mathrm{C}\right)$ results as the difference of the temperature dependence of resistivity at constant strain (TC $\rho)$ and the temperature dependence induced by strain. These two terms are half a order larger (if one does not consider the case $T C R \approx 0$ ) than the difference itself.

2) In spite of the TCR, TC $\rho$ is always negative for the pastes and temperature range considered.

3) In all models, claiming to explain the temperature dependence of TFRs, thermal expansion is neglected (cf. for instance ${ }^{1}$ ). Hence, in these models TC $\rho$ is considered. If such models are compared with experiments, then that has to be taken into account. Thus, the temperature $T_{\min , R}$, at which the resistance reveals its minimum, must be replaced by the corresponding quantity for $\rho$ at $\epsilon=$ const. The difference $T_{\min , \rho}-T_{\min , R}$ of these iwo temperatures is given by:-

$$
\mathrm{T}_{\min , \rho}-\mathrm{T}_{\min , \mathrm{R}} \approx \frac{1}{\rho} \frac{\partial \rho}{\partial \epsilon} \frac{3 \alpha_{\mathrm{s}}}{2 \mathrm{c}}
$$

where $c$ is the parameter introduced by $C$. Marshall ${ }^{7}$ in order to describe the $R(T)$ behaviour of TFR. By means of (3), and the published $\mathrm{GF}_{\mathrm{L}}$ and $\mathrm{c}^{7}$ one obtains:-

$$
\begin{aligned}
& \mathrm{T}_{\min , \rho}-\mathrm{T}_{\min , \mathrm{R}} \approx(440 \ldots 570) \mathrm{K} \text { for } \mathrm{DP} 1441 \\
& \mathrm{~T}_{\min , \rho}-\mathrm{T}_{\min , \mathrm{R}} \approx(350 \ldots 470) \mathrm{K} \text { for DP } 1451
\end{aligned}
$$

The differences are high, because any thermal expansion is suppressed by considering $\rho^{-1} \cdot \partial \rho /\left.\partial \mathrm{T}\right|_{\epsilon=\text { const. }}$

4) All results, concerning microscopic parameters necessary to explain temperature dependence of TFRs (e.g activation energy, ratio of grain resistance/boundary resistance) in the model of reference 1 have been obtained by means of the TCR. Because these

TABLE I

TC $\rho$ of DP 1441 paste

\begin{tabular}{lrrrr}
\hline $\mathrm{T} /{ }^{\circ} \mathrm{C}$ & -25 & 25 & 75 & 125 \\
\hline $\mathrm{TCR} / 10^{-6} \mathrm{~K}^{-1}$ & -71 & 6 & 63 & 103 \\
$\left.\mathrm{TC} \rho\right|_{\max } \mid 10^{-6} \mathrm{~K}^{-1}$ & -330 & -260 & -200 & -160 \\
$\left.\mathrm{TC} \rho\right|_{\nu \mathrm{f}}=\nu_{\mathrm{S}} \mid 10^{-6} \mathrm{~K}^{-1}$ & -420 & -340 & -280 & -220 \\
\hline
\end{tabular}

TABLE II

TC $\rho$ of DP 1451 paste

\begin{tabular}{lrrrr}
\hline $\mathrm{T} /{ }^{\circ} \mathrm{C}$ & -40 & 0 & 40 & 80 \\
\hline $\mathrm{TCR} / 10^{-6} \mathrm{~K}^{-1}$ & -92 & -39 & 3 & 45 \\
$\left.\mathrm{TC} \rho\right|_{\max } \mid 10^{-6} \mathrm{~K}^{-1}$ & -380 & -320 & -280 & -240 \\
$\left.\mathrm{TC} \rho\right|_{\nu_{\mathrm{f}}=\nu_{\mathrm{S}}} \mid 10^{-6} \mathrm{~K}^{-1}$ & -480 & -420 & -380 & -340 \\
\hline
\end{tabular}


data contain the strain-induced temperature dependence $\left(\mathrm{cf}^{1,3}\right)$, such estimations should be repeated with the estimated TC $\rho$-values.

5) For low sheet resistivities the corrections are not small. Assuming $\mathrm{GF}_{\mathrm{L}} \approx 6$, the TCR-value would have to be corrected by a term $100 \cdot 10^{-6} \mathrm{~K}$, which is again of the order of TCR.

6) The same critical remarks are valid regarding the interpretation of low temperature measurements. ${ }^{6}$ The discussion of such experiments becomes still more difficult, because the coefficients of thermal expansion are not constant at low temperatures, not even approximately.

7) Although the quantity $\mathrm{TC} \rho=\rho^{-1} \cdot \partial \rho /\left.\partial \mathrm{T}\right|_{\epsilon=\text { const. is very important from a theore- }}$ tical point of view $\left(\mathrm{cf}^{4,5,6}\right)$, it cannot be determined experimentally. Also if one prepares TFR-samples, which are not supported by a substrate, these samples exhibit thermal expansion and the temperature coefficient of their resistance is given by:-

$$
\left.\mathrm{TCR}\right|_{\text {not supp. }}=\mathrm{TC} \rho+3 \frac{1}{\rho} \frac{\partial \rho}{\partial \epsilon} \alpha_{\mathrm{f}}-\alpha_{\mathrm{f}} .
$$

The temperature at which the resistance of such samples exhibits a minimum is also different from that for TFRs fired on substrates. But the difference of $T_{\min }$-values is now $\sim\left|\alpha_{\mathrm{f}}-\alpha_{\mathrm{s}}\right|$ and becomes much smaller than the values discussed in 3).

8) Because $\alpha_{f}$ depends weakly on $R_{\square}$, the well known empirical relation between $R_{\square}$ and TCR also holds for $R_{\square}$ and TC $\rho$.

In conclusion it should be remarked, that in deriving (2) and (3) it has been assumed that:-
i) $\rho$ is isotropic
ii) $\rho$ remains isotropic if a strain is applied.

The second, very remarkable property follows from the experimental result, that the longtudinal and transversal gauge factors obey the relation $\mathrm{GF}_{\mathrm{L}}-\mathrm{GF}_{\mathrm{T}}=2\left(1+\nu_{\mathrm{s}}\right){ }^{4}$

\section{REFERENCES}

1. G.E. Pike, C.H. Saeger, "Electrical properties and conduction mechanism of Ru-based thick-film (cermet) resistors" J. Appl. Phys. 48, 5152 (1977).

2. M. Prudenziati, "Conduction mechanism in thick-film resistors" in Proc. 3rd Europ. ISHM Conference, Avignon p. 1 (1981).

3. C. Canali, D. Malavesi, B. Morten, B. Prudenziati, A. Taroni, "Piezo-resistive effects in thickfilm resistors" J. Appl. Phys. 51, 3282 (1980).

4. C. Canali, D. Malavesi, B. Morten, M. Prudenziati, A. Taroni, "Strain sensitivity in thick-film resistors", IEEE Transact. on Comp., Hybr., and Manufact. Techn. CHMT-3, 421 (1980).

5. A. Cattaneo, L. Pirozzi, B. Morten, M. Prudenziati, "Influence of the substrate on the electrical properties of thick-film resistors" in Proc. 2nd Europ. ISHM Conference, Ghent p. 123 (1979).

6. P.F. Carcia, A. Ferretti, A. Suna, "Particle size effects in thick-film resistors"J. Appl. Phys. 53, 5282 (1982).

7. C. Marshall, "TCRs: A new approach" in Proc. 2nd European Hybrid Microelectronics Conf., Ghent p. 141 (1979).

8. L.D. Landau, E.M. Lifschitz, Lehrbuch der Theoretischen Physik, Bd. VII, Elastizitätstheorie, Akademie-Verlag, Berlin 1966. 

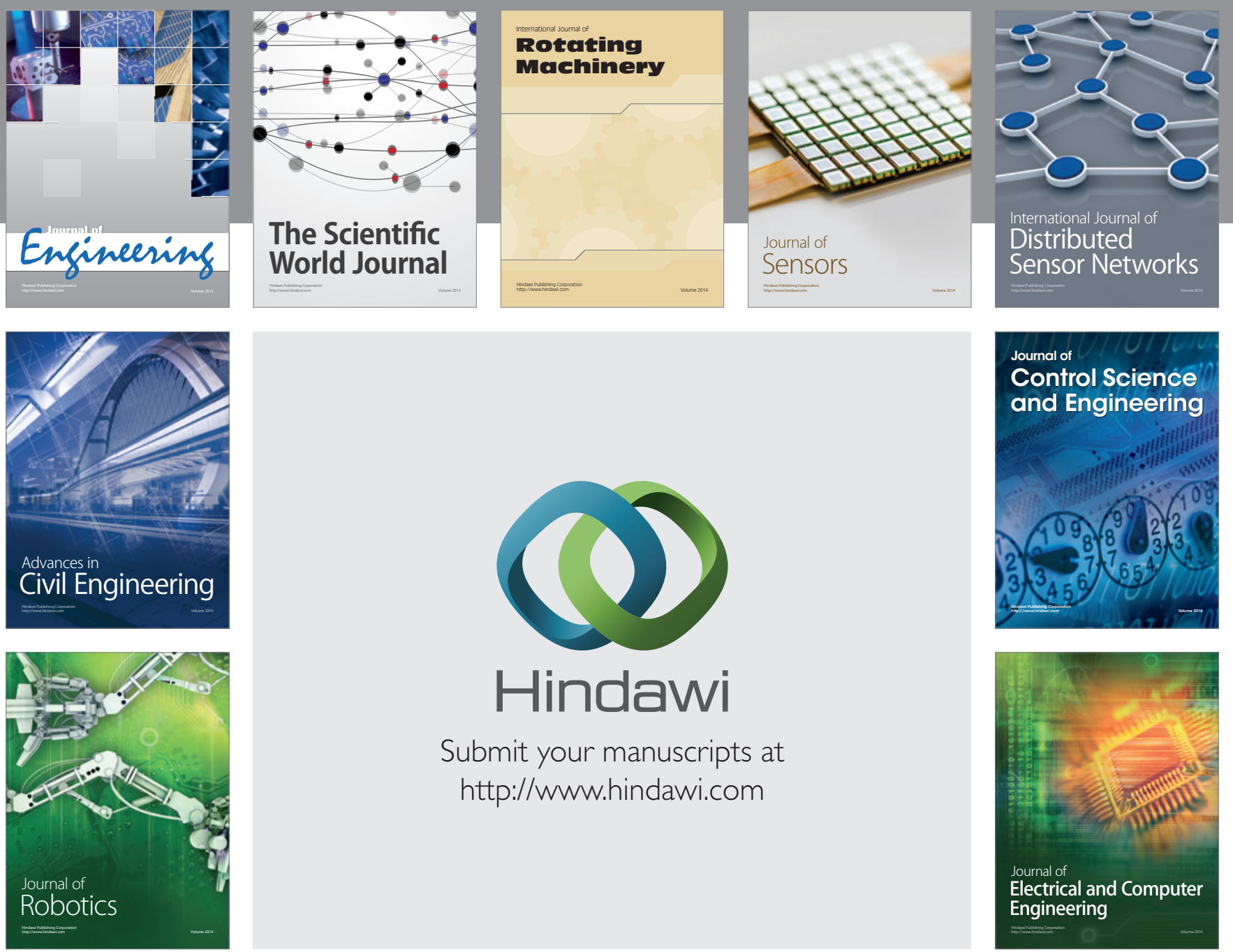

Submit your manuscripts at

http://www.hindawi.com
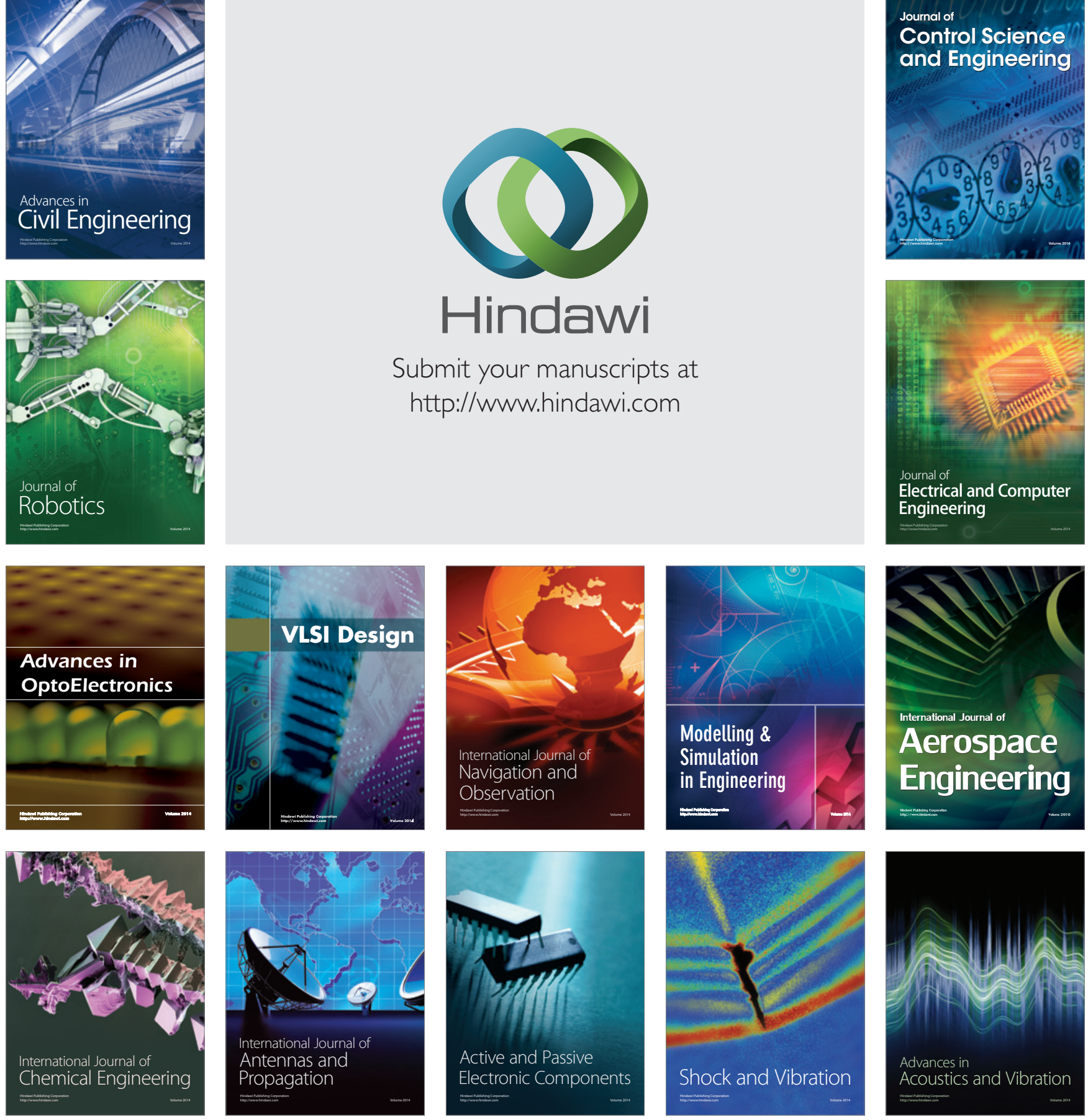